\title{
The Application of Fuzzy Adaptive PID Control in Molecular Distillation Devices
}

\author{
Yue $\mathrm{Wu}^{1, \mathrm{a}}$, Jian Fang ${ }^{1, \mathrm{~b}}$ and Fang Shao ${ }^{1, \mathrm{c}}$ \\ ${ }^{1}$ Jilin Teachers'Institute of Engineering and Technology, Changchun,Jilin , China \\ a28841898@qq.com, ${ }^{b} 757314739 @ q q . c o m,{ }^{c} 182089853 @ q q . c o m$
}

Keywords: PID control; Fuzzy adaptive PID control; Matlab simulation

\begin{abstract}
Molecular distillation efficiency is affected by many factors, such as film motor speed, feed temperature, heat conduction oil temperature, feed amount, vacuum degree, etc. This paper mainly discusses the influence of the speed of the scraper motor on the molecular distillation efficiency.AC asynchronous motor has the characteristics of multivariate, strong coupling, nonlinear parameters. The conventional asynchronous motor speed regulation adopts PID adjustment. This paper adopts fuzzy adaptive PID control instead of traditional PID control, and analyzed by the simulation results.
\end{abstract}

\section{Introduction}

Since the emergence of distillation unit in the 1920s, its theory and research technique are developing rapidly, has already occupied an important position in petroleum chemical industry, food industry, medicine industry. In particular, the separation of materials with high boiling point, viscosity and heat sensitivity. As the world is constantly researching and developing. The technology is slowly moving into broader areas. However, at present, the domestic technology is still relatively backward compared with foreign countries, with high cost, high energy consumption, low yield and many other technologies.[1]Moreover, due to the irreversibility of the molecular distillation process, the loss of energy consumption is also larger. According to statistics, the energy consumed by distillation accounts for about $45 \%$ of the whole chemical industry, so it is an inevitable trend to study the process of molecular distillation, which plays a key role in solving energy problems[2].

\section{Design of Double Closed-Loop PID Controller}

Through the analysis of the influence of operation parameters on molecular distillation, The efficiency of molecular distillation is influenced by many factors, which is a complicated process, Only the relationships between the parameters are balanced. It can be effectively purified. This paper focuses on how to control the film motor speed[3].

The conventional method of motor speed regulation is double closed loop control, composed of the rotating speed and current, The main control loop is the speed control, its main function is to stabilize the speed of the motor, the secondary control loop is the current control, It's a part of the main control loop, It has two functions, When one is in starting, braking and overload armature current maintain on the maximum allowed, to adjust the shortest time and the second is speed at a given value fluctuates up and down, according to the size of the voltage value to adjust the value of armature current[4], the structure schematic diagram is as shown in Fig. 1. 


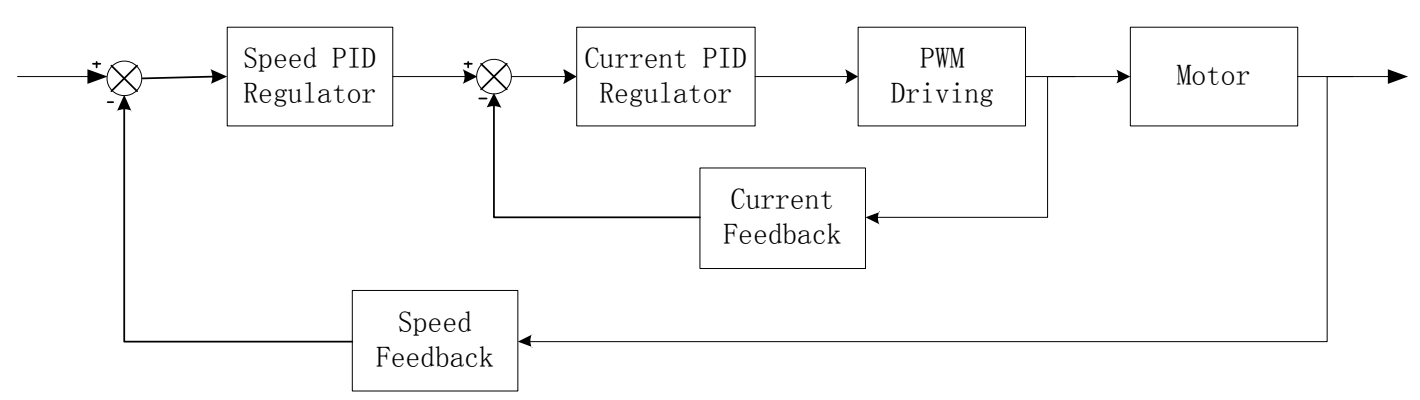

Figure 1. Schematic diagram of double closed-loop speed regulating system

The entire control system uses the output voltage of the speed controller as a given voltage of the current controller. The rotation speed and current double closed loop control are adopted, and two closed-loop controllers adopt PID (PID) control methods. PID is simple, high reliability, is widely used in process control, but the design relies too heavily on the mathematical model of controlled object, and the ability to resist load is not particularly strong, there is a parameter not accordingly along with the change of controlled object shortcomings, when parameters change, the parameters of the PID controller can't make the corresponding adjustment with the change of the controlled object[5].

\section{The Introduction of Fuzzy Control}

Fuzzy control. As the automatic control system gets more and more complicated, The control system has the characteristics of multi-input, multi-output strong coupling, deformation and non-linearity of parameters, Many of the system's mathematical models cannot be established, The advantage of fuzzy control is that there is no need to establish accurate mathematical model of controlled object. Using fuzzy set theory, fuzzy language variables and fuzzy logic reasoning to simulate human thinking. Basic method is: the person of experience with fuzzy conditional statement, said the language variables are quantified using fuzzy set theory, to process the input state with fuzzy reasoning system, thus forming the corresponding control decision, to reduce the overshoot of the system and improve the system response speed, more suitable for time delay and uncertain objects.

A lot of application and experience show that the robustness of fuzzy control is superior to the traditional control strategy, compared with conventional PID, the dynamic response is better, but fuzzy control also exist some disadvantages, once established fuzzy control rules, can't online adjustment, not well adapted to the situation changes, and do not have integral element, thus the steady-state accuracy is not high. Therefore, this paper presents a method to control the speed of the film motor based on the fuzzy self-adaptive PID control, and combines PD, PID and fuzzy control to control the speed ring.

Fuzzy Adaptive PID Control Principle. The ideas of fuzzy adaptive PID control is the fuzzy control theory is applied to control rules, using the fuzzy set said conditions and operating rules, and put the fuzzy control rules are used in computer knowledge base, according to the response of the control system, computer arithmetic of fuzzy reasoning can achieve optimal adjustment of PID parameters automatically. It combines both fuzzy control and traditional PID, traditional PID can not adjust the parameters online, easy to overshoot, the fuzzy adaptive PID precisely to overcome this, namely to improve the dynamic performance and stability of the system, and the large amount of and overcome the overshoot and adjustment time problem[6].Fuzzy parameters self-tuning PID controller adopts two - dimensional fuzzy controller, which combines traditional PID control with fuzzy reasoning, the deviation $e$ and deviation rate $e c$ of the value of the velocity given value $n_{r}$ and the actual velocity value $n_{0}$ are used as the input quantity of the fuzzy controller.And then find the three PID parameters and the deviation and the deviation change rate, the relationship between error in system running continuously, according to the principle of fuzzy control to change of three parameters, in order to meet the different requirements and the controller parameters. 


\section{The Design of the Fuzzy Self-Adaptive PID Controller for Scraper Motor Speed}

Input Ambiguity. Fuzzy controller can only deal with the fuzzy quantity, usually input is not a blur, so you need to do some fuzzy processing, and then do fuzzy reasoning, blurred ultimate aim is to a certain amount into fuzzy reasoning can identify. In the process of russification, the selection of membership function has some influence on the control effect of fuzzy controller. In general, the faster the membership function curve rises, the higher the controller's recognition of the input and output functions. On the contrary, the function curve is relatively stable, indicating that the controller has a stable identification of the input and output functions. Therefore, we must rotate the appropriate membership functions according to the requirements of the system[7].In this paper, it is necessary to choose the triangle membership function with high flexibility to ensure sufficient accuracy when the speed of rotation is achieved without overshoot and stability. Now we define seven fuzzy subset, respectively in the parameters $k_{P} 、 k_{i} 、 k_{d}$, the deviation theory domain $E$ and the deviation change theory $E C$ and at the same time of the seven language value is positive small (PS), the positive medium (PM), positive big(PB), negative medium(NM), negative big(NB), negative small (NS) and negative zero $(\mathrm{Z})$. Using trigonometric functions as a membership function, the input and output values $e, e c 、 k_{p} 、 k_{i} 、 k_{d}$ and the domain of the sum are $\{-3,-2,-1,1,2,3\}$.

Fuzzy reasoning process. After fuzzy processing of the non-fuzzy quantity, the fuzzy reasoning process is to be carried out. Fuzzy reasoning is to use the established fuzzy rules to determine the fuzzy relationship between input and output, then use the established fuzzy relation to control the output. The Fig. 2 is the output curve of the general step response[8].

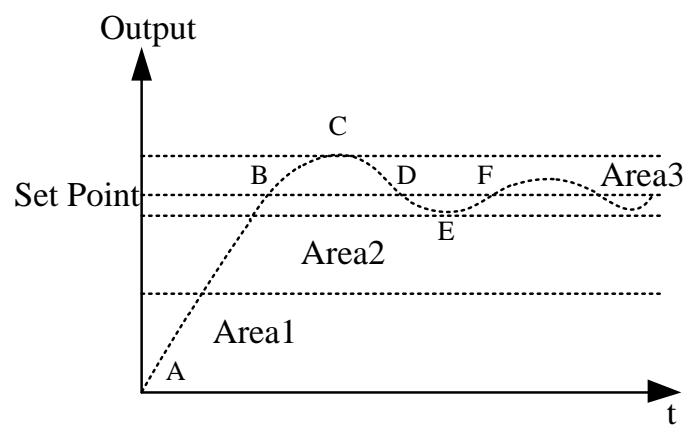

Figure 2. The output curve of the general step response

The output curve is divided into regions 1, 2 and 3, and the important points of A, B, C, D, E and $\mathrm{F}$ are analyzed. According to the law of their change, the corresponding parameters and the fuzzy rules are formulated according to this change.

Solutions of the blur. The fuzzy relation can be obtained by using the method to the parameters and reasoning in the fuzzy rules table.

Fuzzy condition statement:

$$
\text { if } e=a \text { and } e c=b \text { then } y=c
$$

Their fuzzy relationships are as follows:

$$
R_{i}=(a \times b) \times c=(a \times c) \bigcap(b \times c)
$$

$\mathrm{A}$ and $\mathrm{b}$ have the same dimension, and the controller's fuzzy condition statement is as follows:

$$
R=R_{d l} \bigcap R_{b l}
$$

$l=1,2,3 \ldots, \mathrm{N}$. Based on the inference rules and fuzzy relationships, the fuzzy sets and corresponding output sets of the given input are obtained:

$$
c^{\prime}=\bigcup_{l=1}^{N}\left[\left(a^{\prime} \times R_{a l}\right) \bigcap\left(b^{\prime} \times R_{a l}\right)\right]
$$


A collection of fuzzy controller can output variable language reflect the value of complete, elected for controlled object can only use a control volume control, to release an accurate control values from the output subset, this process is called fuzzy processing, this paper uses gravity method for solving fuzzy, formula is as follows:

$$
u(k)=K_{p} e(k)+K \sum_{j=1}^{k} e(j)+K_{d} \Delta e(k) / T
$$

In the formula, $u(k)$ is the output of the $k$ sampling period, $e(k)$ is the output of the $k$ sampling period bias, $T$ is the sampling period for the system. The output $u$ of the system is obtained by the product of the output $u(k)$ and the proportional factor $K_{u}$, The form of $u$ is shown in the formula:

$$
u=u(k) k_{u}
$$

\section{Simulation and analysis of scraper speed control system}

MATLAB is one of the computer aided design tools of the control system, provide an effective toolbox support. We have used the fuzzy toolbox in MATLAB to establish the input and output function and fuzzy inference rules. In addition, dynamic system modeling and simulation tool SIMULINK is embedded in MATLAB[9].SIMULINK has the advantages of simple modeling, easy operation, simple feasibility and convenient modification of system parameters. The simulation results are shown in Fig. 3 and Fig. 4.

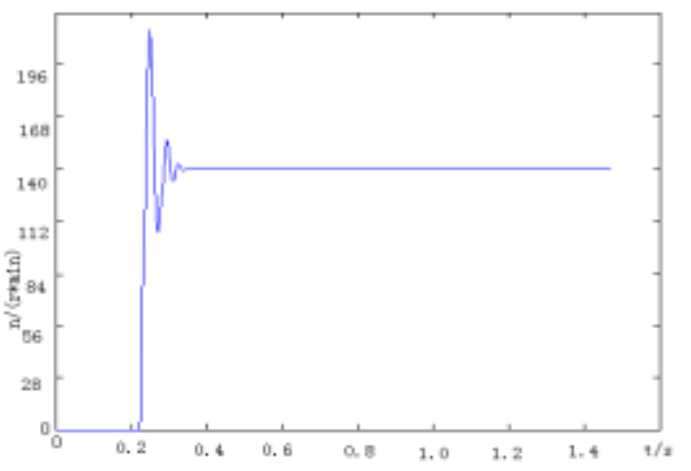

Figure 3. Conventional PID simulation curve

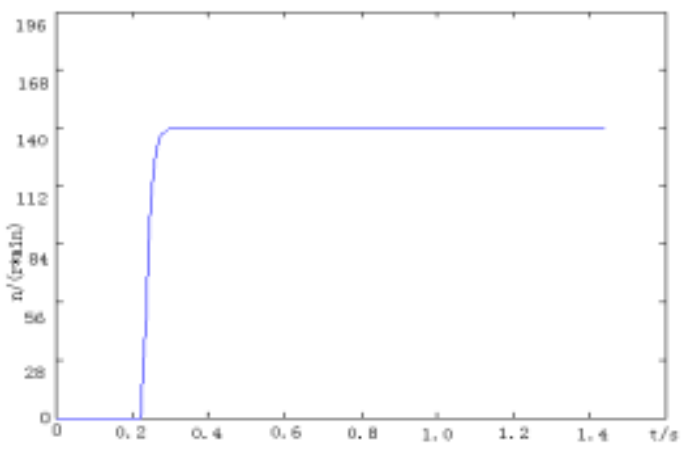

Figure 4. Fuzzy adaptive PID simulation curve

Through the simulation curve can see, the fuzzy adaptive PID than the conventional PID control is more ideal, can in a relatively short period of time to stabilize, overshoot is small, the interference has strong robustness and adaptability, and has good prospects in engineering application[10].

\section{Summary}

On the basis of the conventional PID controller, this paper designed a controller on the basis of the principle of threshold switch, combine conventional PID and fuzzy controller to control speed ring, finally carries on the simulation using MATLAB/SIMULINK, the experimental results show that the fuzzy adaptive PID compared with the conventional PID, with super scale down small, smooth excessive, short setting time, good dynamic response and stability characteristics.

\section{References}

[1] J.H. Lian. Molecular distillation techniques and their applications[J]. Chemical technology and development,2010,39(7):32-38.

[2] F.F.Chen. Molecular distillation and its application in residual oil separation[J]. Petrochemical technology and application,2010,289(3): 253-257. 
[3] J.L.Sun. A brief discussion on molecular distillation techniques[J]. China petroleum and chemical standards and quality, 2012,11(5): 30-32.

[4] X.H. Hua. Numerical simulation and parameter analysis of the mathematical model of the distillation process of thin film evaporator. 2009,25(2): 93-98.

[5] S.Y. Wang. Research on control method based on fuzzy PID controller[J]. Mechanical science and technology,2011,30(1): 166-172.(In Chinese)

[6] S. Mao. Fuzzy-PID design and control performance analysis[J].Journal of China inertial technology,2006,14(2): 60-68.(In Chinese)

[7] J.T. Li. The application of parameter self-tuning fuzzy PID in temperature control[J]. Technical and engineering applications,2007(37). (In Chinese)

[8] N.Shi. The application of fuzzy self-tuning PID control algorithm in molecular distillation unit[D]. Changchun university of technology,2012.

[9] J. Sun. Based on PLC fuzzy control system research[J]. Huadian technology,2009(31): 22-25.(In Chinese)

[10]X.M. Shi. Fuzzy control and MATLAB simulation[M].Tsinghua university press,2008.(In Chinese) 